\title{
BOBINA DE TESLA MUSICAL
}

Francimar Conceição dos Santos'; Larissa Vanile Santos Carneiro"; Michell Thompson Ferreira Santiago ${ }^{2}$; Rafael Maia Gadelha ${ }^{1}$; Tiago Jairo Vieira de Almeida ${ }^{1}$; Carlos Augusto F. de Jesus Ribeiro²; Tiara Borges Santiago Ferreira3;

1 Faculdade Metropolitana de Camaçari - Tv. da Avenida Leste; Camaçari/BA; francimar.tec@hotmail.com

2 Universidade Federal da Bahia; R. Prof. Aristídes Novis, 2 - Federação, Salvador/BA;

${ }^{3}$ Universidade Cândido Mendes; Campos Rua Anita Peçanha, 100 - Pq. São Caetano - Campos dos Goytacazes/RJ

Resumo: A fundamentação deste trabalho consiste na reprodução de um projeto já existente na literatura em um modelo simples sem aplicação didática para aprendizagem; tendo como objetivo, inseri-lo ao ambiente acadêmico por meio das aulas práticas na disciplina de circuitos elétricos para os discentes do curso de Engenharia de Controle e Automação. Sendo apresentado a eles o conhecimento teórico das funcionalidades de cada peça ou dispositivo inserido na estrutura do projeto através de explicações baseadas em teorias existentes; auxiliando-os durante a sua execução. Foi obtido êxito na sua funcionalidade, mas constataram-se algumas instabilidades; superaquecimento de alguns componentes e até mesmo danos irreversíveis nos mesmos sendo necessário à sua substituição por novos.

Palavras-Chave: Plotter; Arduino; CNC; Tubos PVC.

\section{TESLA COIL MUSIC}

\begin{abstract}
The basis of this work is the reproduction of an existing project in the literature in a simple model without didactic application for learning; aiming to insert it into the academic environment through practical classes in the discipline of electrical circuits for students of the Control Engineering and Automation course. Being presented to them the theoretical knowledge of the functionalities of each piece or device inserted in the project structure through explanations based on existing theories; assisting them during their execution. Its functionality was successful, but some instabilities were found; overheating of some components and even irreversible damage to them requiring replacement with new ones.
\end{abstract}

Keyswords: Plotter; Arduino; CNC; PVC duct. 


\section{INTRODUÇÃO}

A bobina de Tesla ou transformador ressonante foi construída por Nikola Tesla no fim do século XIX, de acordo com o Instituto de Física da Universidade de Brasília, o projeto original utilizava uma bobina de 12 milhões de volts e suas descargas elétricas possuíam 38 metros de extensão, entre dois eletrodos colocados a uma altura de 61 metros do solo.

O experimento consiste em uma bobina de tesla que conta com o auxílio de uma placa de Arduino que tem como função controlar a frequência da corrente elétrica que transita pelo equipamento proporcionando a emissão de ondas sonoras perceptíveis ao ouvido humano executando sequências lógicas por meio da mesma que se assemelham a toques polifônicos.

Inserindo-o ao ambiente educacional por meio de uma experimentação prática; relacionando o mesmo aos conhecimentos teóricos discutidos em sala, promovendo uma maior compreensão da teoria $\mathrm{x}$ prática que também pode ser aplicado em outras instituições educacionais por possuir um baixo custo financeiro sendo que a grande parte do material pode ser reaproveitado do lixo eletrônico para a sua confecção e não se faz necessário uma grande estrutura laboratorial para executar a sua montagem.

Para a execução do experimento, os discentes consultaram sites da internet, utilizando como base o conteúdo disponibilizado no site do professor Marlon Nardi Walendorff [1], acrescentando algumas alterações para melhorar a qualidade sonora e da estrutura.

\section{METODOLOGIA}

Aplicando os conhecimentos teóricos adquiridos na disciplina de Circuitos Elétricos, foi desenvolvido um dispositivo eletrônico durante as aulas no período da segunda unidade do semestre, onde pode ser aplicada atividades práticas com peso avaliativo, das 72 horas/aulas disponíveis da disciplina, foi utilizado o tempo de 4 horas semanais por três semanas consecutivas, onde aproximadamente 20 alunos dividiram-se em equipes para realização da montagem do dispositivo. Os discentes utilizaram a linguagem de programação $C$, para realizar toques polifônicos através da utilização do arduino interligado a bobina de Tesla. Com isso foi possível reproduzir de forma polifônica a música Sweet Child O' Mine, cuja programação foi disponibilizada no site do professor Marlon [1], porém para fins expositivos, foi acrescentado um loop à programação, fazendo-a tocar repetidamente. Logo o leque metodológico esteve assentado no processo formativo e expositivo.

Dando partida a descrição das etapas de construção e desenvolvimento do experimento citado, iniciando-o com a reutilização de materiais presentes em equipamentos existentes no laboratório da instituição de ensino Faculdade Metropolitana de Camaçari (Famec), devido ao êxito obtido na execução e funcionamento do projeto o mesmo foi exposto em um evento aberto ao público dentro das instalações da faculdade o (Encontro Cientifico da Famec - Encif) para cerca de 100 pessoas em um auditório. 
A escolha deste tipo de ambiente consiste por questões de interferências externas na acústica conservando uma melhor audição durante a sua execução; deste modo não se faz presente a necessidade de um alto falante ou qualquer dispositivo eletrônico por conta que a intensidade sonora seria suficiente para que todas as pessoas escutassem a música.

Neste experimento foram aproveitados componentes eletrônicos que iriam ser descartados entre eles: monitores CRT (Cathodic Ray Tube) de onde foi removido o flyback, placas de televisores, fontes de computador, diodos da placa dos televisores, transistor bc548 de fontes, resistores de fontes, condutores de cobre com espessura de $0,5 \mathrm{~mm}$ encontrados em cabos de rede, a placa de madeira de um armário que tinha sido rejeitado, o dissipador de calor foi retirado de uma antiga fonte de computador, a fonte de alimentação aproveitamos de um switch que tinha queimado exceto $\mathrm{o}$ arduino que nós compramos.

\subsection{Descrições Dos Componentes}

\subsubsection{Arduino UNO R3}

Em conformidade com Thompson, em termos práticos, um Arduino é um pequeno computador que você pode programar para processar entradas e saídas entre o dispositivo e os componentes externos conectados a ele. O Arduino é que chamamos de plataforma de computação física ou embarcada, ou seja, um sistema que pode interagir com seu ambiente por meio de hardware e software [2].

\subsubsection{Flyback}

Segundo Braga (1996) o transformador de saída horizontal (TSH) conhecido como flyback pelo seu princípio de funcionamento tem por função gerar a alta tensão que acelera os elétrons no cinescópio da ordem de milhares de volts e o sinal dente de serra para a varredura horizontal [3].

\subsubsection{Transistores}

Em conformidade com Boylestad (2013) o transistor é um dispositivo semicondutor de três camadas que consiste em duas camadas de material do tipo $\mathrm{N}$ e uma do tipo P ou em duas camadas do tipo P e uma do tipo N [4].

\subsubsection{Resistores}

Segundo Nilson e Riedel (2009) a resistência é a capacidade dos materiais de impedir o fluxo de corrente ou, mais especificamente, o fluxo de carga elétrica. O elemento de circuito usado para modelar esse comportamento é o resistor. Como conceito, podemos entender a resistência se imaginarmos os elétrons que compõem a corrente elétrica interagindo com a estrutura atômica do material no qual estão se movimentando, a qual, por sua vez, resiste a eles [5]. 


\subsubsection{Fonte de Alimentação}

De acordo com Nilson e Riedel (2009) uma fonte elétrica é um dispositivo capaz de converter energia não elétrica em energia elétrica e vice-versa. Quando uma pilha se descarrega, converte energia química em energia elétrica, ao passo que, quando ela se carrega, converte energia elétrica em energia química [5].

\subsubsection{Condutores}

De acordo com Gebran (2017) o material condutor conduz bem a eletricidade, pois tem uma estrutura que permite que elétrons se movam livremente. Ainda assim sempre haverá alguma perda de energia [6].

\subsubsection{Dissipador de Calor}

Conforme Hart (2012), um dissipador de calor reduz a temperatura da junção para uma dada dissipação de potência de um dispositivo pela redução da resistência térmica total da junção. $O$ encapsulamento do dispositivo é sempre fixado ao dissipador de calor com uma pasta térmica para preencher alguns vazios entre as imperfeições das superfícies do encapsulamento e do dissipador. Existem dissipadores de calor disponíveis em várias medidas, que podem ser desde pequenos dispositivos com presilhas até perfilados maciços de alumínio extrudado [7].

\subsection{Lista De Materiais}

1 - Flyback modelo:AT2079/B9 retirado de um monitor de PC de tubo;

1 - Transistor 2N3055;

1 - Transistor BC548B;

1 - Resistor de 1K / 1/4W (marrom, preto, vermelho, dourado);

2 - Resistores de 15R / 1/4W (marrom, verde, preto, dourado);

1 - Arduino UNO R3;

1 - Cabo para o Arduino;

1 - Fio de espessura $1,5 \mathrm{~mm}^{2}$ e comprimento $43,5 \mathrm{~cm}$ cor vermelha;

1 - Fio de espessura $0,5 \mathrm{~mm}^{2}$ e comprimento de $48,5 \mathrm{~cm}$ cor preta;

1 - Fio de espessura $0,5 \mathrm{~mm}^{2}$ e comprimento de $25 \mathrm{~cm}$ cor vermelha;

1 - Fio de espessura $0,5 \mathrm{~mm}^{2}$ e comprimento de $25 \mathrm{~cm}$ cor preta;

2 - Jumpers para protoboard macho e aproximadamente $15 \mathrm{~cm}$;

2 - Pedaços de arame de espessura de $0,89 \mathrm{~mm}$ e comprimento de $34 \mathrm{~cm}$;

2 - Parafusos soberbos de espessura $4 \mathrm{~mm}$ e $14 \mathrm{~mm}$ de comprimento;

1 - Base de retangular de madeira;

1 - Fonte de corrente contínua 12VDC/5A.

1 - Dissipador de calor

1 - Cooler modelo: 40×40×10mm

1 - Pasta térmica 


\subsection{Lista De Ferramentas}

Ferro de solda;

Estanho;

Pistola de cola quente;

Alicate de corte;

Alicate de bico;

Extrator de solda.

\subsection{Ilustrações}

Figura 1. Circuito eletrônico da bobina de tesla musical com Arduino

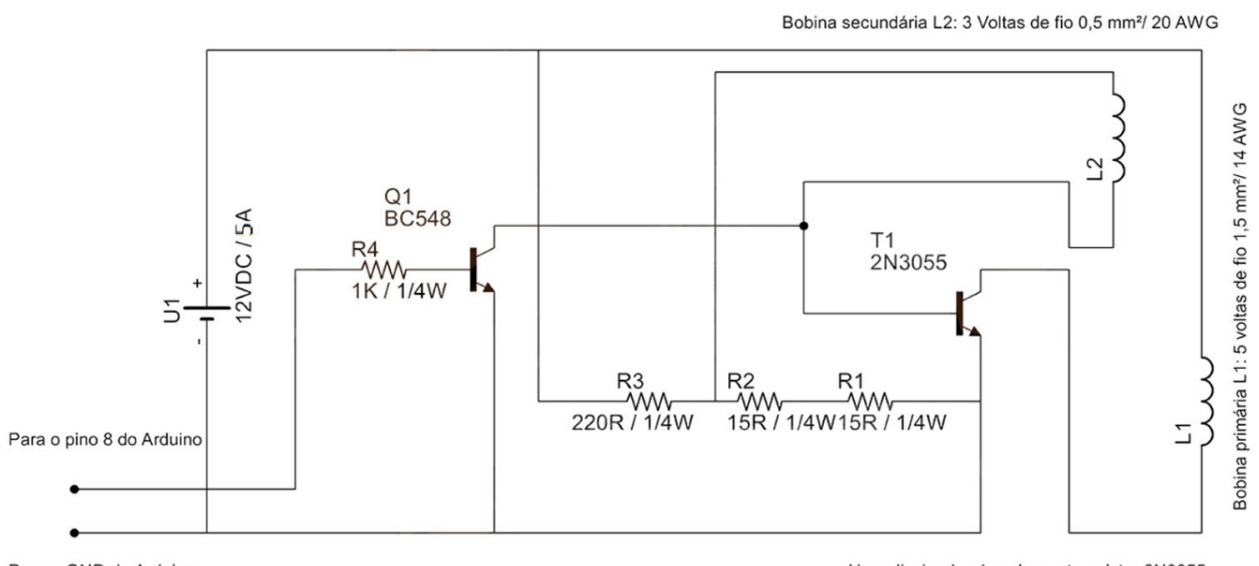

Figura 2. Terminais do Transistor NPN 2N3055

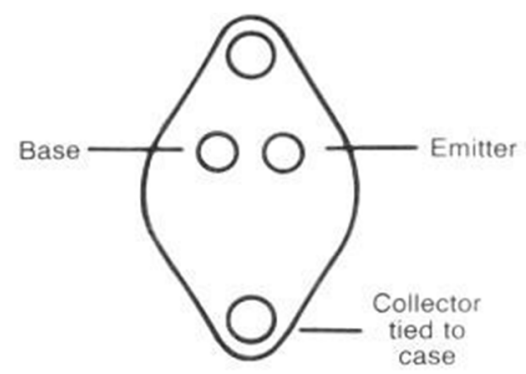

Figura 3. Terminais do Transistor NPN BC548
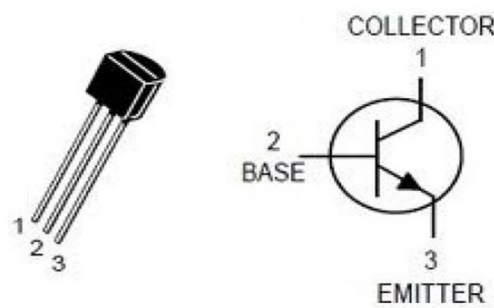

BC 548 Transistor 


\section{RESULTADOS E DISCUSSÃO}

A partir do desenvolvimento e testes do equipamento após o seu funcionamento, constatou-se a necessidades de melhorias na sua estrutura física devido a algumas eventualidades. Foram realizadas modificações pontuais no projeto inicial, devido a falhas que provocavam superaquecimento no transistor chegando a queimar o mesmo e movimentação das hastes de arame galvanizado que não possuíam estabilidade.

Figura 4. Bobina em Funcionamento.

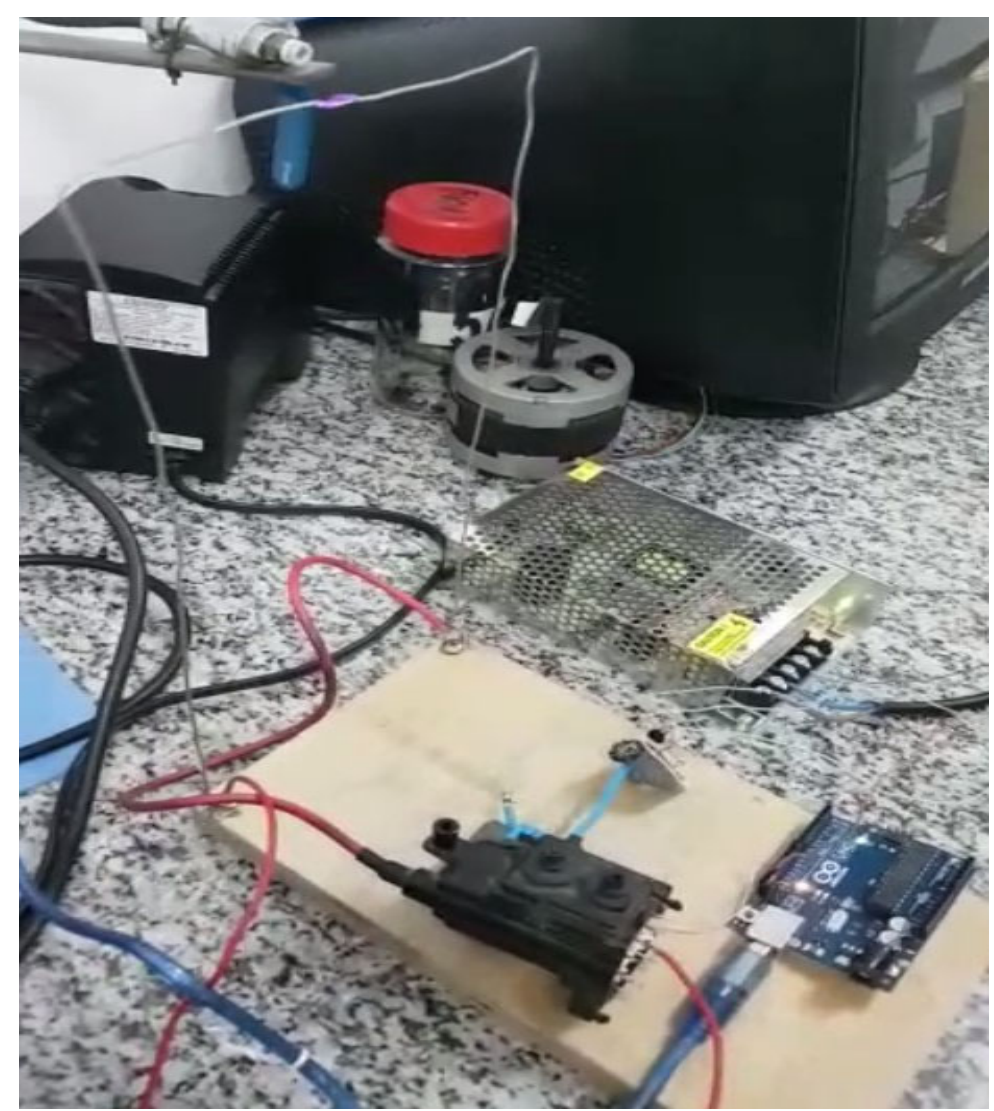

Por consequência foi criado um suporte com um polímero ABS (Acrylonitrile Butadiene Styrene) impresso na impressora 3D, com o objetivo de aumentar a aderência das hastes para quando o circuito for ligado não vibrar com a tensão elétrica, que passa cerca de $3000 \mathrm{v}$, foi inserido um cooler ao dissipador de calor acoplado ao transistor, entre o dissipador e o transistor foi aplicado uma camada de pasta térmica promovendo um resfriamento mais eficiente ao equipamento; na programação dos toques foi acrescentado um loop mantendo a sua execução permanente. 


\section{CONCLUSÃO}

Em virtude do que foi proposto na inserção deste experimento, no ambiente educacional, a prática proporcionou aos discentes que participaram diretamente da construção do dispositivo e aos que apenas desfrutaram da sua exibição no ENCIF uma experiência autêntica do que realmente é uma aplicação prática. Direcionando o interesse destes alunos a tomarem novas iniciativas relacionadas ao aproveitamento de materiais que seriam descartados e aplicando-os em novos dispositivos que podem ser desenvolvidos por eles mesmos dentro ou fora das escolas e instituições de nível superior.

\section{REFERÊNCIAS}

[1] WALENDORFF, MARLON NARDI. Projeto - Bobina de tesla que toca músicas com arduino e Flyback, circuito explicado. Disponível em <https://www.marlonnardi.com/p/bobina-de-tesla-que-toca-muiscas-com.html/>. Acesso em: 8 de abr. de 2019.

[2] THOMPSON, M. Arduino Básico. São Paulo. NOVATEC Editora, 2011.

[3] BRAGA, N. C. Trabalhando com fly-backs (ART885). Disponível em: $<$ https://newtoncbraga.com.br/index.php/eletronica/52-artigos-tecnicos/artigosdiversos/7252-trabalhando-com-fly-backs-art885/> Acesso em:10 Jul. 2019.

[4] BOYLESTAND, R. L.; NASHELSKY, L. Dispositivos Eletrônicos - Teoria de Circuitos. Tradução de Sonia Midori Yamamoto. 11. ed. São Paulo. 1993. Pearson Education do Brasil.

[5] NILSSON, J. W; RIEDEL, S. A; Circuitos Elétricos. Tradução de Arlete Sinille Marques. 8. ed. São Paulo. 2009. Pearson Prentice Hall.

[6] GERBRAN, A.; RIZZATO, F. Instalações Elétricas Prediais. Porto Alegre. Bookman, 2017.

[7] HART, D. W. Eletrônica de Potência: Análise e Projetos de Circuitos. Porto Alegre. Amgh Editora, 2012. 